\title{
The Planeterrella experiment: from individual initiative to networking
}

\author{
Jean Lilensten ${ }^{1}{ }^{*}$, Gabrielle Provan ${ }^{2}$, Sandrine Grimald ${ }^{3}$, Asgeir Brekke ${ }^{4}$, Erwin Flückiger $^{5}$, Petra Vanlommel ${ }^{6}$ \\ Cyril Simon Wedlund ${ }^{7,8}$, Mathieu Barthélémy ${ }^{1}$, and Pierre Garnier ${ }^{3}$ \\ 1 Institut de Planétologie et d'Astrophysique de Grenoble, 38041 Grenoble, France \\ ${ }^{*}$ Corresponding author: jean.lilensten@obs.ujf-grenoble.fr \\ 2 Institution University of Leicester, LE1 7RH Leicester, UK \\ 3 Onera - The French Aerospace Lab, 31055 Toulouse, France \\ 4 History of Geoph. and Space Sciences Journal, University of Tromso, Department of Science and Technology, \\ Faculty of Science and Technology, N-9037 Tromso, Norway \\ 5 Physikalisches Institut, University of Bern, Sidlerstrasse 5, 3012 Berne, Switzerland \\ 6 Royal Observatory of Belgium, Rue Groeselenberg 57, 1180 Uccle, Belgium \\ 7 Belgian Institute for Space Aeronomy, Brussels, Belgium \\ 8 Now at Finnish Meteorological Institute, Helsinki, Finland
}

Received 4 June 2012 / Accepted 1 February 2013

\section{ABSTRACT}

\begin{abstract}
Space weather is a relatively new discipline, which is still largely unknown amongst the wider public despite its increasing importance in all of our daily lives. Outreach activities can promote awareness of space weather. In particular the visual beauty and excitement of the aurora make these lights a wonderful inspirational hook to enhance understanding of space weather in a general audience. A century ago, the Norwegian experimental physicist Kristian Birkeland, one of the founding fathers of modern space science, demonstrated with his Terrella experiment the formation of the aurora. Recently, a modernized version of the Terrella has been designed. This "Planeterrella" experiment allows the visualization of many phenomena that occur in our space environment. Although the Planeterrella was originally a local project, it has developed to become a very successful international public outreach experiment. We believe that its success is due to mainly two factors (i) the Planeterrella is not patented and the plans are free to any public institute and (ii) the project is widely advertised using national and European scientific networks such as COST ES 0803, as well as press releases, books and web sites. Today, seven Planeterrellas are in operation, four more are under construction in four different countries and several more are being planned. During the last five years, about 50000 people in Europe have attended live Planeterrella demonstration on the formation of auroral light, the space environment and space weather. Many more have seen the Planeterrella being demonstrated on TV. The Planeterrella received the first international prize for outreach activities from the Europlanet Framework 7 program in 2010 and the French Ministry of Science outreach prize "Le goût des sciences" in November 2012. This paper describes the process that led to the construction of the first Planeterrella and discusses how the Planeterrella project developed to become an international public outreach phenomenon. We also examine some of the lessons learnt along the way.
\end{abstract}

Key words. outreach - aurora - experimental

\section{Introduction}

This paper reports on an auroral simulator called the Planeterrella. The Planeterrella is made of a vacuum chamber in which electrons are fired at a magnetized sphere. The electrons become trapped on magnetic field lines and create a radiation belt surrounding the sphere. Some of the electrons are guided to the two poles of the magnet where they interact with the remaining gas in the chamber to produce glowing "auroral" ovals. The glow produced is due to the de-excitation of the remnant gas in the chamber. The word "vacuum" designates here a primary vacuum, i.e., with a pressure of a few Pascal; this is sometimes referred to as "rarefied gas".

The first Planeterrella was built in 2008 with the support of different agencies and networks (see the Acknowledgements section). Over the last 5 years the Planeterrella has been a very successful public outreach project, with several copies now in operating in different European countries. The aim of this paper is to examine the initiation and the development of this successful project. Please note that this paper does not intend to be a scientific paper or to be a detailed guide for reproducing the experiment. Some of the physics behind the Planeterrella has been described in Lilensten et al. (2009).

The paper commences with a description of the Planeterrella experiment including the constraints of the projects, the cost and the funding. Then we analyse the strengths and weakness of the project. Finally, we show how this experiment, only designed to be a popular science exhibit, can also be used for teaching, scientific modelling and art.

\section{The Terrella}

Kristian Olaf Birkeland (1867-1917) was born in Kristiania (now Oslo) where his father was a storekeeper and a shipowner. He became interested in natural sciences at a very young age and it is claimed that the first thing he bought for himself was a magnet. Before he was 18 he had written an important 
paper demonstrating his exceptional skills in mathematics. From 1885 he studied at the Royal Frederiks University of Kristiania. He graduated in 1890 as a teacher of the natural sciences (Egeland \& Burke 2005).

During his studies, Birkeland became fascinated by physics. As soon as he had completed his final university examination, he became involved in a study of "electrical oscillations in a thread of metal". In 1893 Birkeland received a scholarship that gave him the opportunity to carry on his studies and visit foreign universities and research groups. First he travelled to Paris and met the well-known physicist Henri Poincaré (1854-1913) who had made pioneering work in calculating the trajectories of charged particles in a magnetic field. While in Paris Birkeland became interested in the motion of cathode rays (i.e. electrons) in magnetic fields. He performed experiments on this subject.

From Paris, Birkeland went to Genève where he met Professor Lousien de la Rive (1834-1924), the son of the late Professor Auguste-Arthur de la Rive (1801-1873) who had studied electric discharges in rarefied gases and formulated a theory about the aurora borealis as a discharge phenomenon. Lousien de la Rive, however, together with Edouard Sarasin (1843-1917) introduced Birkeland to the electromagnetic waves discovered by Heinrich Hertz (1857-1894) in 1888 .

When Birkeland came back to Kristiania, he performed several experiments with different discharge tubes and magnets to observe how cathode rays behave close to magnetic poles. He noticed that the electric discharges were bent and drawn to the poles where they created fluorescence illumination. In 1896 Birkeland published his theory about the aurora, where he postulated that it was caused by cathode rays streaming out from the Sun, captured by the Earth's magnetic field and forced to converge towards the magnetic poles where they collided with molecules and atoms in Earth's atmosphere forming a fluorescent ring around each pole: aurora borealis and aurora australis. Figure 1 is a popular picture of $\mathrm{K}$. Birkeland at his apparatus.

In 1901 Birkeland built a new set of laboratory experiments using magnetized spheres to simulate cosmic phenomena, these were developed in response to criticisms of his auroral theory. At first he named the device a spherical electromagnet, but later on he used the term Terrella or "little Earth". Between 1901 and 1906 he constructed cylindrical and spherical vacuum chambers. Probably around 1909 he commenced designing box-shaped chambers with straight glass walls, the smallest was $22 \mathrm{~L}$ in volume while the largest was referred to as the "1000 L prismatic chamber" or "the Universe". Altogether Birkeland developed 17 different vacuum chambers (Fig. 2). The technical specifications of Birkeland's experiments were as follows: the "vacuum" was actually about $10 \mathrm{~Pa}$, the voltage was larger than $300 \mathrm{~V}$ for a current on the order of $0.1-1.0 \mathrm{~mA}$ and the intensity of the magnetic field was approximately $0.5 \mathrm{~T}$ at $0.5 \mathrm{~cm}$ from the surface of the magnets. These conditions, though easily achievable today, posed far greater challenges at the turn of the 20th century.

\section{The Planeterrella}

\subsection{Rebuilding the Terrella}

In 1996 Terje Brundtland, an engineer from Tromsø in Norway, restored the largest original Terrella built by Birkeland, called "The Universe" (Brundtland 1997). Due to the historical importance of this experiment, original components were used

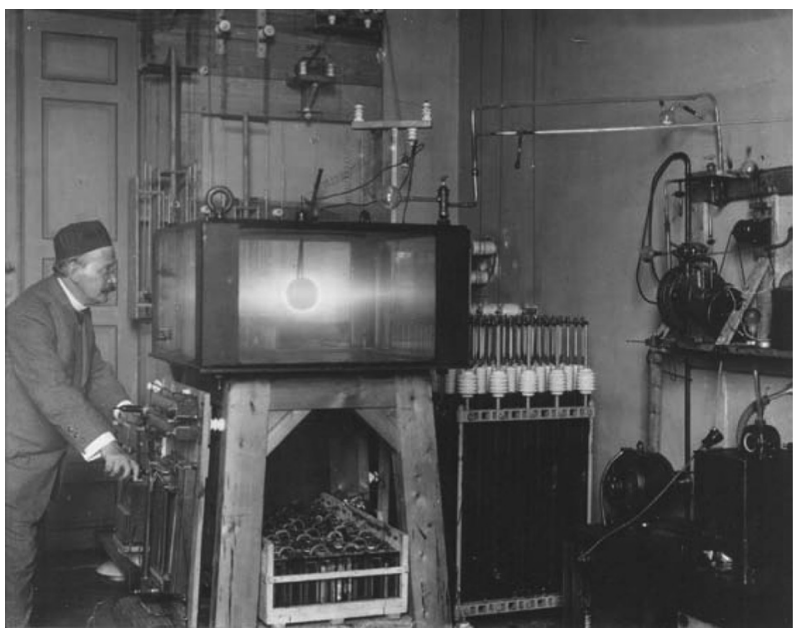

Fig. 1. Professor Kristian Birkeland at one of his vacuum chambers performing Terrella experiments.

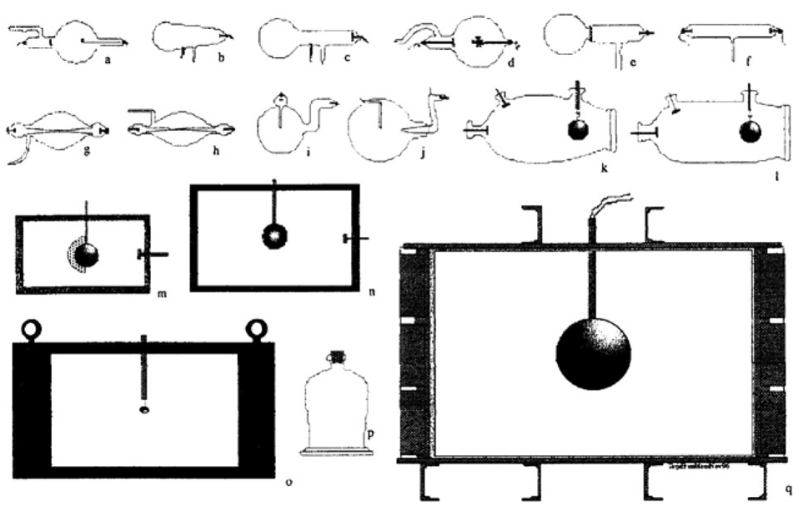

Fig. 2. The 17 vacuum chambers used by professor Birkeland for his experiments related to the aurora borealis and other cosmic phenomena. In all these designs, the sphere was suspended. The electric duct is seen in some of these drawings as a bar crossing the chamber (i.e. Terrella $\mathrm{n}$ and $\mathrm{m}$ ). Birkeland did not draw it every times (i.e. Terrella $\mathrm{o}, \mathrm{p}$ or $\mathrm{q}$ ).

whenever possible. However, safety, budget and ease-of-maintenance also had to be taken into account. The glass was changed to a more modern variety, and some of the original wooden supports that were in particularly poor conditions had to be re-built. The restoration was completed in the late 1990's and the restored Terrella has become a popular exhibit at the Auroral Observatory in Tromsø.

After visiting the Auroral Observatory, we built several Terrellas. Amongst these, one was only built due to the drive of an enthusiastic high school physics teacher together with some of his students. It received a silver medal at the "Olympics of Physics". Another one was assembled during an international school held at ICTP in Italy (Messerotti et al. 2008). Based on these experiences, three co-authors of this paper (JL as the initiator, CSW and $\mathrm{MB}$ ) and a team of collaborators who are acknowledged at the end of this paper improved and modernized the experiment. This new experiment is firmly based on the Terrella. However, it is more flexible, more powerful and allows one to visualize many more planetary phenomena that $\mathrm{K}$. Birkeland could with the original Terrella. We decided to call it the "Planeterrella", a name that gives tribute to the Terrella while still suggesting the new planetary features. 


\subsection{Planeterrella I}

We considered several ways of improving the Terrella. Firstly, Birkeland hung the magnetized spheres. Such a suspension has an inherent fragility as the attachment may break. Moreover, if the sphere is suspended it is not possible to add a second sphere, as the two magnetized spheres will attract or repel each other, resulting in the potential snapping of the attachments. This is why Birkeland's successive Terrellas only grew in size, not in configuration. In the improved design the spheres are placed on supports enabling several spheres to be placed in the chamber simultaneously. We also endeavoured to make the Planeterrella design as flexible as possible, so that a number of different cosmic phenomena can be visualized. In Birkeland's original experiment there was a simple piece of metal for the electron source which also exists in the Planeterrella in order to re-create Birkeland's observations. However, this duct can now move along a support, and the height of the spheres above the surface of the vacuum chamber may be also varied (Fig. 3).

These modifications allowed many more space science phenomena to be visualized than originally observed by Birkeland. It has even been possible to conjecture the sunward auroral observations at Uranus and predict the shape of its night side auroral oval (Lilensten et al. 2009; Gronoff \& Simon-Wedlund 2011).

\subsection{Goals and constraints}

Our main goal was to improve the Terrella in order to provide the best possible auroral demonstration. We were mindful to respect Birkeland's original experiment, firmly rooting our new experiment in his original design. Our original target audience were groups of local people such as school classes of all ages, adults and the amateur astronomers. Our maximum target audience number was about 30 . The size of the experiment was restrained by the size of the medium-sized car in which the experiment would be transported. Finally, the experiment had to be assembled and dismantled without tools. The Planeterrella was designed within these constraints.

The completed vacuum chamber is $50 \mathrm{~L}$ in volume and $47 \mathrm{~cm}$ in diameter. The two aluminium spheres (Birkeland used copper) have a diameter of 10 and $5 \mathrm{~cm}$. The Planeterrella is designed to be operated by a professional scientist or engineer. The electrical power supply is the same as in university student laboratory and is earthed. The thickness of the vacuum chamber is such that it cannot explode and cannot be easily broken. The gases from the vacuum pump are evacuated through an exhaust tube. For a fully automated Planeterrella (not operated by a scientist or engineer), many more safety constraints would have to be considered.

\subsection{Costs and funding}

The main cost was clearly the time spent designing and building the Planeterrella, we estimate that about $200 \mathrm{~h}$ was spent designing the Planeterrella and that it took about $160 \mathrm{~h}$ to build. The experimental equipment cost approximately EUR 8000 in France. However, it may be possible for others wishing to replicate the Planeterrella to source much of the equipment, such as the vacuum pump and the power supply, within universities so that the actual cost can decrease to less than EUR 3000. This was the estimated cost of the University of Orléans, which built part of its Planeterrella within the university's workshop. This is a relatively small amount of money compared to other scientific

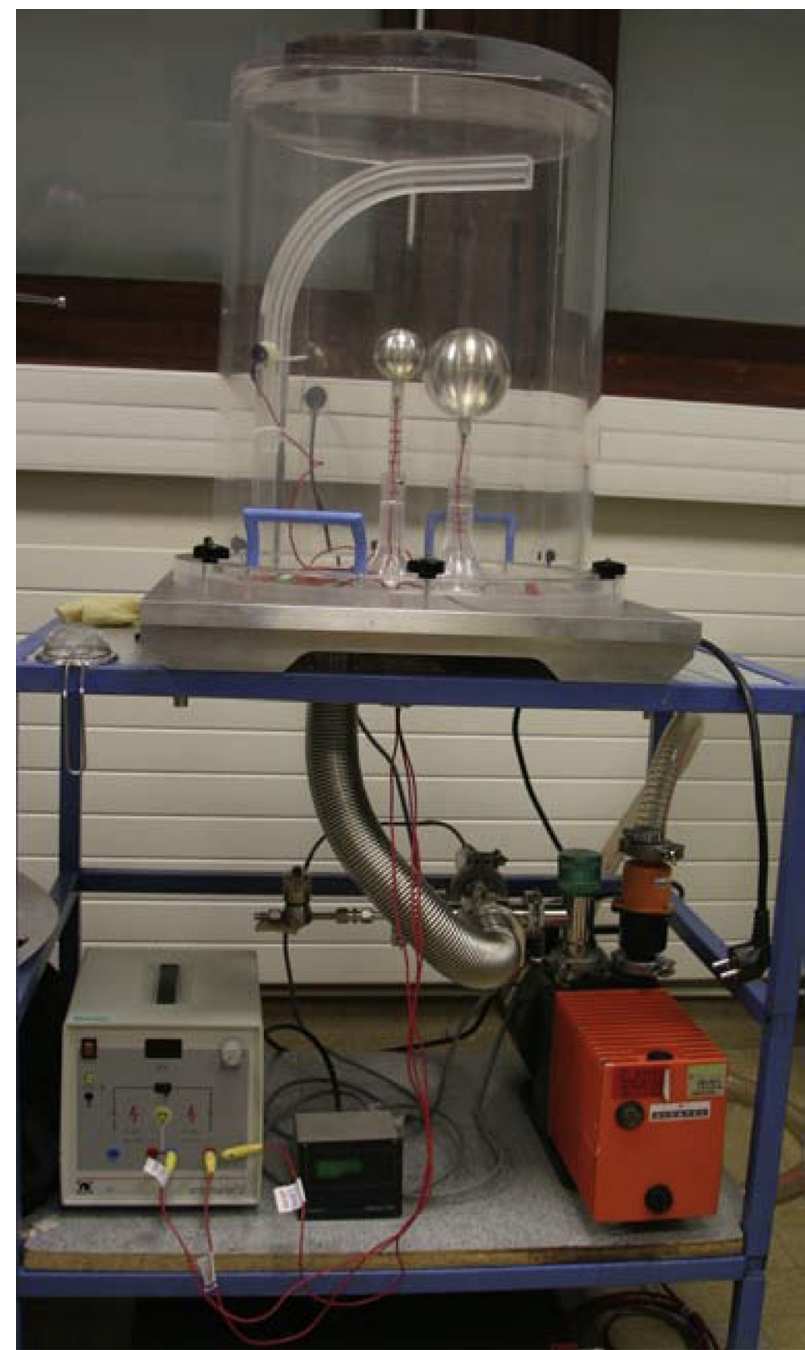

Fig. 3. View of the Planeterrella I. The vacuum chamber is made from Plexiglas. Its external diameter is $47 \mathrm{~cm}$. The spheres (respectively 5 and $10 \mathrm{~cm}$ of diameter) are positioned on Plexiglas supports. The electric duct is positioned horizontally on the left of the picture on the bended Plexiglas arm. On the lower plate of the demonstration table, there is a power supply (white box on the left), a manometer monitor (black box in the middle) and a vacuum pump (orange, on the right).

experiments, but in our European countries this still represents a significant sum to spend on outreach activities.

Each country has its own funding streams and structures. In France, if funding had had to be secured in advance through a call and selection by a committee, the Planeterrella would never have been funded. The original Planeterrella was actually built using money from research contracts related to space weather without being explicitly mentioned in the research proposals. Naturally, it was fully described in the progress reports. None of the funding agencies complained about this expenditure. This is not always possible in other countries where the funding is provided through answers to calls. Now that the experiment has proven to be successful, other institutes find it less difficult to secure funding.

\subsection{Planeterrella II and future prospects}

After 2 years of presenting Planeterrella I at public outreach events and venues, we decided to build a new Planeterrella. We wanted to be able to move the spheres in order to produce 


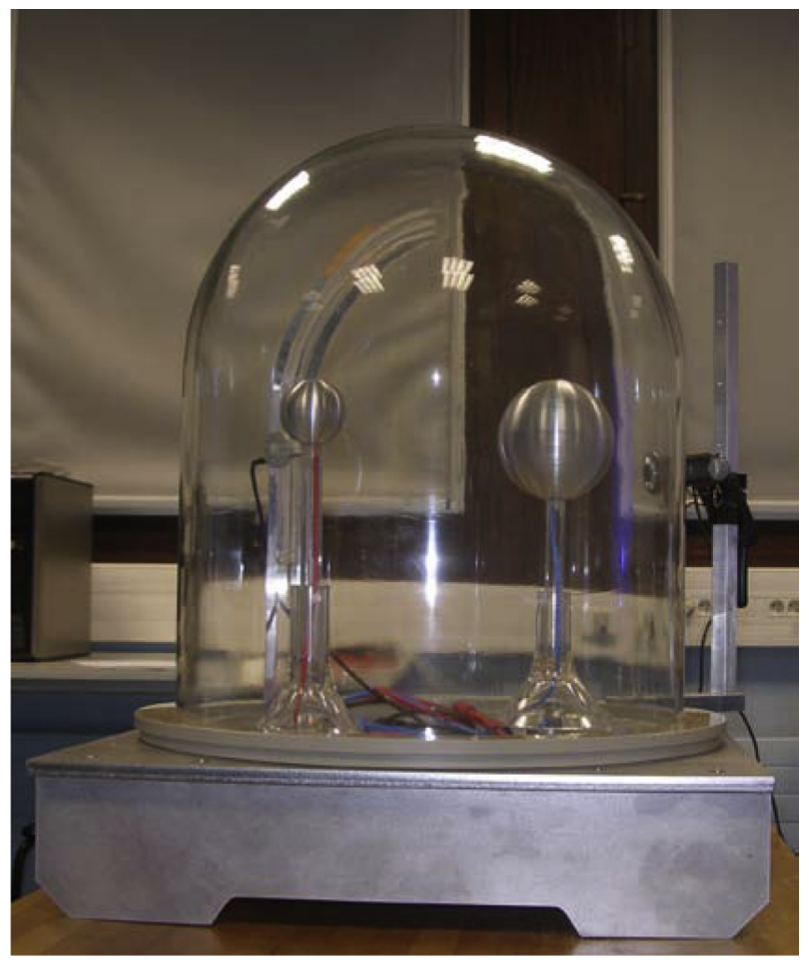

Fig. 4. View of the Planeterrella II. The vacuum chamber is in glass. The supports of the spheres are made such that they can move. For this purpose, the plate supporting the experiment had to be raised. On the right side, a small camera is hooked on a simple support. This support can rotate around the vacuum chamber. The external diameter of the vacuum chamber is $415 \mathrm{~mm}$.

a more spectacular auroral display. The Plexiglas vacuum chamber had also become somewhat opaque due to UV emissions. We decided to have a vacuum chamber made of glass. Unlike Plexiglas, it is possible to make a bell-shaped glass vacuum chamber, allowing a better view from all directions and from above. The vacuum chamber could be filled with other gases than air. A set of reference lines was drawn on the base of the chamber in order to facilitate measurements.

These improvements were incorporated in Planeterrella II, which was completed in October 2011 (Fig. 4). The movable spheres allow one to picture the bow shock in front of the sub-solar magnetopause when the spheres are moved towards each other (although the public must be warned that the real bow shock originates in a more complex mechanism involving ions as well).

A third version of the Planeterrella was completed in November 2012. Planeterrella III includes a camera. The main reason for this is that the Planeterrella has become so popular that it is often shown to audience exceeding 100 people. Another motivation comes from a new artistic development (see below). In Planeterrella III, the spheres can rotate so one can visualize auroral ovals rotating with the tilted magnetic field (in this way it also becomes clearer that the mechanism is constrained by this magnetic field) or the stellar corona moving with the star (Fig. 5). In the longer term, improvements will include the use of electromagnets instead of permanent rare earth magnets. The pace of these developments largely depends on funding.

\subsection{Small vs. big}

The actual size of the experiment has been constrained by the factors described above. The size of the Planeterrella prevents

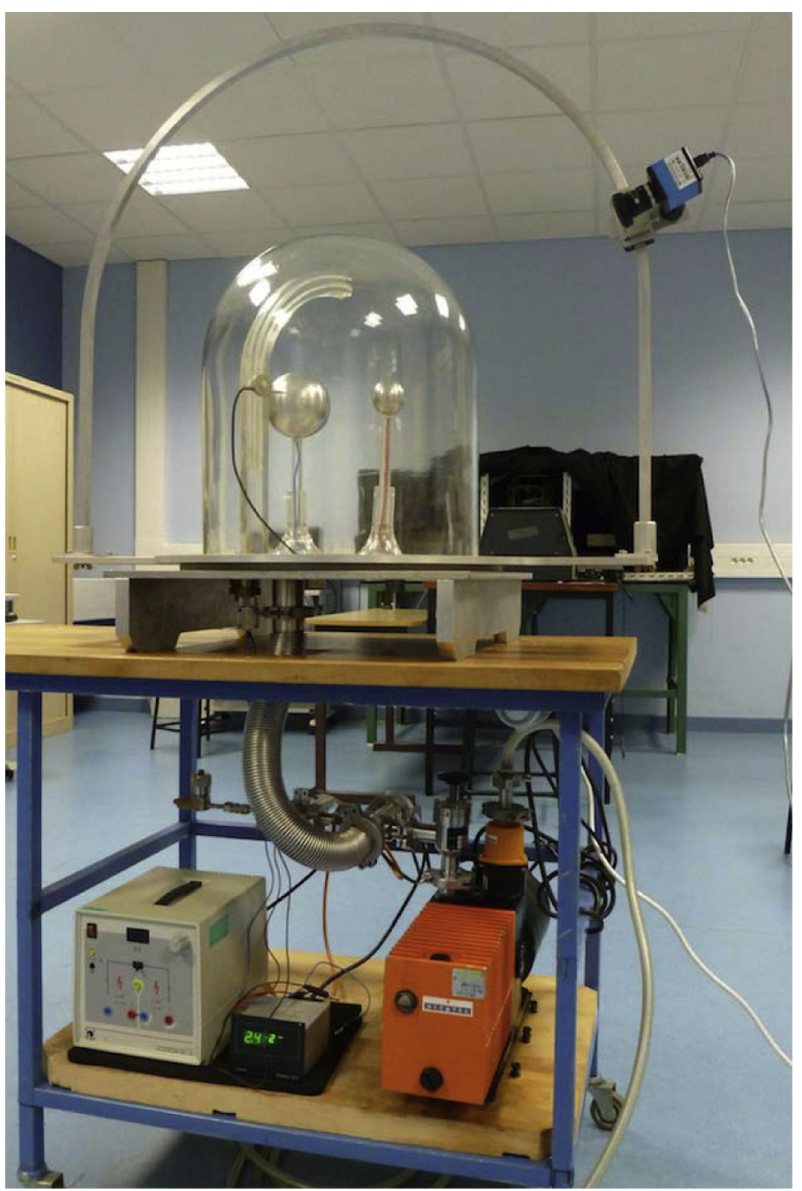

Fig. 5. View of the Planeterrella III. The camera is now hooked on an inverted-U support. The support can rotate around the vacuum chamber and the camera can move freely on this support. The supports of the spheres have been improved to allow now the spheres to rotate and not only to move forward. Here, the pressure is $2.4 \times 10^{-2} \mathrm{mb}$.

it from being demonstrated to a large audience without a camera and a video projector. However, such an intimate exhibit has also many advantages. The close proximity of the audience to the exhibit may allow the audience to forget that they are looking at spheres. As soon as the darkness falls, many feel that they are gigantic people in space looking at real stars and planets. A wide variety of audiences have reacted with a child-like awe to the Planeterrella: students and pupils of all ages, families, people from the country and the cities, psychologists, scientists, two Space Agency presidents and the president of the CNRS. This is the advantage of having a small and more intimate exhibit.

\subsection{Automated vs. operated}

The main advantage of an automatic experiment is that it can be exhibited in museums. The French space museum (Cité de l'Espace in Toulouse) tried to automate the Planeterrella. Their attempt failed due to the significant increase in health and safety considerations, which could not be met by the Planeterrella. For example, it is important that the spheres don't fall down if anybody hits the demonstration table, and nobody must be hurt by accidentally touching the pump or the tubes. An automated Planeterrella would therefore have to be very static, and indeed only a Terrella (not a Planeterrella) has been successfully automated. A private company made the Terrella for Tromsø 


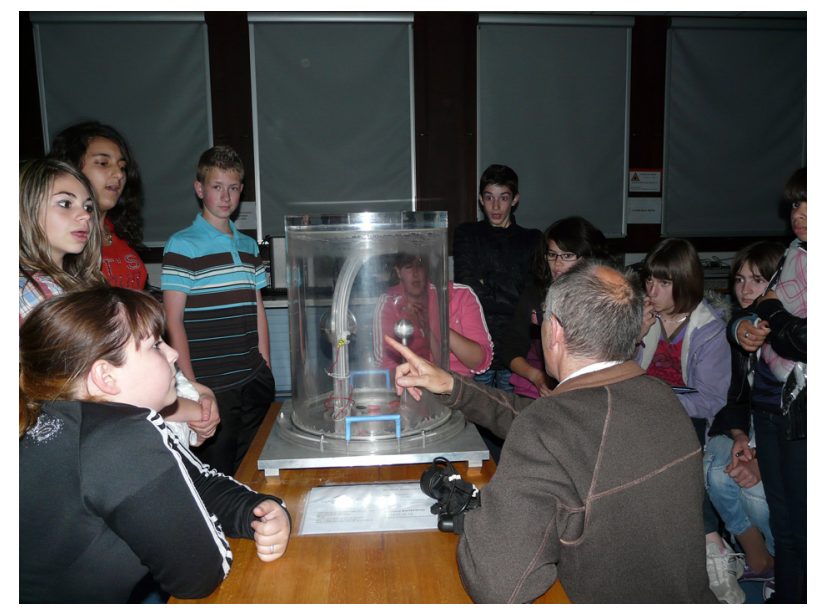

Fig. 6. A demonstration with high school students.

Museum in Norway. Having a manned experiment has other advantages not linked to flexibility. The most important and maybe surprising is that it looks new and modern to visitors who are used to numerical and robotic demonstrations, rather than physical and manually operated experiments. This is certainly the main lesson learned from this experiment. It also provides an opportunity to engage in a scientific dialogue with the general public, answering questions and queries on space sciences. Of course, information posters may answer some questions, but no poster can answer in advance all the questions. Moreover, some people cannot read (about $9 \%$ of France's population are illiterate) but are still curious about science (Fig. 6).

\subsection{Gentleman's agreement vs. patent}

When the Planeterrella was created, the CNRS and the University suggested patenting it. This was refused for a variety of reasons. Firstly, Birkeland did not patent the original Terrella (although he patented his industrial inventions). Secondly, the philosophy underlying this new experiment is that public science is costly but the productions of the public science should remain free for the public. Our view is that our work has already been paid by the tax-contributors, and there is no reason to get a second contribution. The French administrations (CNRS and University) agreed not to patent the Planeterrella. Instead, CNRS prepared a "Gentleman's Agreement" to be signed by the two parties. If the other party is a public institute such as a university or a museum then CNRS provides all the plans and support needed to build a working Planeterrella free of charge. A private company has to pay the institute. The other party agrees not to share the plans with others, to share any improvements and to acknowledge the author of the experiment and his laboratory when exhibiting the Planeterrella. Scientists are used to networking and sharing ideas, and informal collaborations have been vitally important in disseminating information about the Planeterrella.

\section{Networking: sharing and mitigation}

In order to introduce the Planeterrella to a wide audience, it is important to advertise it. However, public relations and communication are not the core business of European researchers. Europe is lagging behind countries such as the US, where scientific institutes do have extended advertisement programmes. The Planeterrella web site (http://Planeterrella.obs.ujf-grenoble.fr) was created with a small budget. The most powerful tool however, which enables European initiatives to survive and spread, is networking. Through a French solar-terrestrial scientific network (PNST), the experiment was shown to the French space weather community. This resulted in three Planeterrellas being built in France, while four additional are under preparation. In Europe, COST ES 0803 and the Europlanet FP7 actions have been excellent arenas through which to spread information on the Planeterrella, and it has resulted in Planeterrellas in the UK and Switzerland. Furthermore, two Planeterrellas will soon be completed in Belgium, one in Spain and one in the Langley NASA center. An agreement has been signed with Italy and with Norway. The University of California, Los Angeles (UCLA) heard of the Planeterrella through these networks and signed the agreement as well.

Several signed agreements not mentioned above have failed to result in a working Planeterrella. On every occasion the reason was the lack of technicians and scientists committed to making the experiment work. In one case, a Planeterrella was built but since no experimenter was available, it remains in a cabinet. Although the plans and the tricks to make the experiment work are provided, building and demonstrating the Planeterrella is a matter of time and availability. A lack of funding is not the reason for failure, a lack of manpower is.

Altogether, we estimate that the seven existing Planeterrellas have resulted in 50000 people viewing live Planeterrella demonstrations. This number does not include the many viewers who have seen the Planeterrella on TV shows. In France, it has been displayed twice on the national television channel (Antenne 2), in the most popular scientific show with an approximate audience of 5 million viewers. The second broadcast was for the Christmas special edition. In Germany, it was shown in a movie about Birkeland on the ZDF channel. Below we present three separate Planeterrella case studies. Two of the Planeterrellas are operational, one in the UK and the other in Switzerland. The third one concerns a project in Brussels, Belgium that aims to adapt the design.

\subsection{The Leicester example}

In 2010/2011 Dr. Gabby Provan and Dr. Alan Stocker from the University of Leicester won a Science and Technology Facilities Council (STFC) Small Award to purchase and display a Planeterrella. The Planeterrella is the only one in the UK.

The original aim was to engage and educate a total of about 1000 visitors in the wonders of solar-terrestrial and space physics by using the visual beauty and excitement of the aurora as observed on Earth and other planets. The Planeterrella forms the centrepiece of a number of "Auroral Shows". These shows include a demonstration of the Planeterrella, a presentation of the latest STFC-supported auroral research and a Questions and Answers session. The "Auroral Show" are staged at local schools and colleges, at the University of Leicester (e.g. during open days and school visits) and in other community settings. It was originally anticipated that 10 shows would be held during the course of the 12-month period of the project, the majority of these to be directed at school pupils in the age range 11-18 years.

In order to facilitate the teaching of the Planeterrella within the British school system, additional support was requested from the research council which allowed Leicester to employ qualified teachers to produce lesson packs which link the Planeterrella to the British National Curriculum. Four lesson 
packs have been produced, providing all the material a scientist or engineer needs for a 1-h presentation of the Planeterrella to Key Stages 2, 3, 4 and 5 (ages 7-18). The lessons "stand alone" and each pack contains a lesson plan as well as additional materials such as magnets, "mini-magnetospheres" (a magnet with iron filings suspended in silicon oil), handouts etc. The packs also include a teacher's guide that can be sent to the school before the visit.

All in all the Planeterrella has proved to be a very successful public outreach project and has been exhibited to about 25000 visitors. The space lesson packs have been taught at five schools and have been very well received. The Planeterrella has been publicized through a host of channels, which included writing to individual schools and, to the Science, Technology, Engineering and Mathematics Network (STEMNET). In addition to the STFC Small Award, the University of Leicester won an Institute of Physics University School Link Award, which paid for students to assist in demonstrating the Planeterrella. Although the original STFC Small Award and the Institute of Physics award project periods are now finished, the Planeterrella project is still very much ongoing with almost weekly requests for Auroral shows. In 2012 the Planeterrella was exhibited at the British Science Festival in Aberdeen and at the Cheltenham Science Festival, to a combined potential audience of tens of thousands.

\subsection{The Bern example}

The Planeterrella project in Bern, Switzerland, was a joint venture between a grammar school (Gymnasium Lerbermatt) and the Space Research and Planetary Sciences Division at the University of Bern. It was initiated in 2010 by Mathieu Dufour, a student who was looking for a fascinating subject for a thesis project required for the school leaving examination. Under the lead of Dr. Ingo Leya, and in close collaboration with the school teachers Dr. Hans Kammer and Mrs. Irma Mgeladze, the University built two copies of Planeterrella I. Engineers and a construction team that usually build space experiments were involved in the project. A shared cost plan was established for the items that had to be purchased (e.g., Plexiglas vacuum chamber and vacuum pumps). Apprentices in the division's mechanical workshop manufactured most of the other parts, and progress of the work was documented in a movie. The Planeterrella at the University was a key attraction of the space physics booth during the "Night of Science" in Bern in September 2011, an event intended to bring science and scientists closer to the general public. More than 7000 visitors attended this event. The apparatus is now integrated in the physics department's collection of demonstration experiments, which are used in lectures and public outreach activities. Mathieu Dufour participated in the 2012 Swiss National competition of "Schweizer Jugend forscht" with the school's version of the Planeterrella, For his presentation about Northern Lights, he got the Metrohm special award and an invitation for the 9th Expo-Sciences Europe 2012 (ESE 2012) in Tula, Russia. Thus, the Bern Planeterrella project was extremely successful, and it will continue to be a key attraction and demonstration experiment for a broad audience.

\subsection{The STCE example}

This is an example where the initial Planeterrella has been modified and adapted for a slightly different purpose. The Solar-Terrestrial Centre of Excellence (STCE), a Belgian science and application centre, is building its own Planeterrella experiment. The STCE provides the financial and human resources. The original Planeterrella was only designed for educational purposes, STCE want to modify the Planeterrella so that it can also be used for scientific experiments. The scientific aims drive specific requirements; the experiment needs to be mobile and easy to operate, to have a flexible and adaptable configuration, and to be safe for the operator and audience. The cost of the Planeterrella is budgeted.

With the educational and scientific goals in mind, a team of scientists, technicians and communication officers was allocated to the task. The STCE decided to build two experiments: a prototype based on the original Planeterrella experiment and a second experiment, which had additional functionalities. The scientists came up with the following list of suggestions for improvement:

1. Rotating spheres: the two spheres represent the Sun and the Earth. If the Earth can rotate around its axis (simulation the Earth's daily rotation), then viewers can observe that the auroral ovals do not rotate with it.

2. Electromagnets in the spheres: the electromagnets represent the dipolar magnetic field of the Sun and the Earth. Electromagnets allow the strength of the magnetic field to be varied. If a coil is placed around the bar magnet, then a broad range in magnetic field strengths from 0 Tesla up to $\sim 1.5$ Tesla can be achieved. If the electromagnets rotate, then one can simulate a polarity flip similar to how the Sun's magnetic field flips every 11 years. Allowing the tilt between the electromagnet and the rotation axis of the sphere to vary can contribute to a realistic configuration. To simulate active regions and sunspots, we can add a small electromagnet near the surface of the large sphere (i.e. the Sun). This will visualize the coronal loops.

3. Simulation of the Dawn-Dusk Electric field: this field is transversal to the direction of the Sun-Earth line. Extra large electrodes are necessary to create this additional E-field. A conductive plate as electrode hinders the view. The electrode could be also a conducting grid.

4. Changeable conductivity of the spheres: if the sphere is a conductor then it can act as an electron gun. The electrons leave the conducting sphere and are guided by the potential/E-field and the magnetic field to the other sphere. The Earth's ionosphere is a varying conductor: the dayside has a larger conductivity than the night side. Is it possible to change the conductivity of the spheres?

By developing a prototype, the STCE has been able to perform a feasibility study of the functionalities proposed by the scientists involved in the experiment.

The technical plans for the prototype were drawn up and companies contacted. For the prototype, the STCE has chosen a vacuum chamber made from Plexiglas. Plexiglas is lighter than glass, and thus it is will be easier to transport the experiment. Plexiglas is less UV transparent compared to glass. Plexiglas polarizes light, allowing scientists to perform experiments light polarization. The vacuum chamber is a cylinder with a flat top. The work to meet the scientific and educational objectives will start as soon as the prototype will be completed.

The scientific objectives will focus on measuring auroral emission lines at different wavelengths. For this purpose, different gases will be injected into the vacuum chamber. As the bell jar is made of Plexiglas, the auroral emission lines will be 
polarized. The polarization will depend on the strength of the magnetic field. The Planeterrella will allow us to verify certain theoretical objectives. The experiment allows one to check the degradation of filters caused by UV radiation. This could be of value for space-based experiments like PROBA2/SWAP and LYRA.

The Planeterrella will also be used for educational purposes. The Planeterrella allows space science to be presented in an interactive way between the educator and the audience. We define five potential target audiences. Each target audience needs a specific approach:

- The non-professional and non-scientific community includes the general public, local institutes, schools and press. These users have to be addressed in their own language with a non-scientific and understandable vocabulary.

- The non-professional but with a strong scientific interest. They have often a large knowledge.

- The group that looks for a science education for professional reasons. We include students both at undergraduate as at PhD level, scientists that are new to the field of space sciences, as well as people involved in the programming and management aspects of a scientific space science project.

- Commercial Entities gather people, companies and institutes that are defined as space weather end users.

- Political entities like governments, national agencies, national science policy, and international political structures are another important target group.

In this communication project, we have to search and contact our target groups. This is why the requirement of the Planeterrella to be mobile is crucial. However, we could think of other techniques and tactics to reach our targets through social media like YouTube. A demonstration could be taped and made available on the Internet. The result is not the same as the real thing, but a movie gives a first idea.

The education capabilities are evident: visualizing and explaining aurora, measuring UV transmission and comparing it with the UV radiation outside. The Planeterrella represents a beautiful and accessible introduction to space science and space weather. Finally, in the spirit of the Gentleman's Agreement, future Planeterrellas will benefit from these advances.

\section{A multipurpose experiment}

The Planeterrella can be utilized for a wide range of uses. The Planeterrella is a popular feature at scientific and artistic exhibitions. It is used as a teaching tool in university physics departments, for example to examine the motion of charged particles in a magnetic field, to study the physics of rarefied gases and to perform spectral analysis. The Planeterrella allows us to visualize astronomical phenomena such as the ring currents circling the planets, the solar corona or auroral ovals. In the RhôneAlpes area of France the Planeterrella has become very popular, with students coming to work on it or use it for in their reports.

\subsection{Pedagogic}

Below we describe a pedagogic project from the Department of Physical Measurements in the Technological Institute of the Toulouse 3 University in 2010/2012. This institute trains future technicians and engineers. During their second year the students have to perform a tutored project lasting several weeks. The project involves solving a real-life practical problem, and then presenting and defending their work. The purpose of the tutored projects is to make the students work independently and in teams.

Two groups of students used the Planeterrella for their projects. Their work was twofold. First they examined the Planeterrella from the perspective of vacuum engineering: magnetism, spectroscopy, measurement chains and the physics of electric discharges. Secondly, they were instructed to improve the experiment such that it could show new phenomena and better represent reality. Before tackling these problems, the students had to understand the experiment: why it was built, which phenomena are shown and how it is constructed. The main pedagogic interest of this tutored project is that the students work to improve an existing experience.

The students decided to concentrate on moving one of the spheres without opening the chamber and thus without breaking the vacuum. They designed a low-cost system, which allowed the small sphere to move easily (experiencing only a small amount of friction). The system, shown in Figure 7, consists of a PVC plate, which moves linearly on two plastic curtain rails. The small sphere is attached to the plate. The movement comes from using two magnets (in orange in Fig. 7). The first one is located below the Planeterrella support. As it is located outside the vacuum chamber, it may move freely. The second one is located above the PVC plate inside the vacuum chamber. The two magnets are magnetically connected to each other. When moving the external magnet, the second one is dragged along. This system only allows translational (back and forth) motion, but such motion is actually less likely to cause malfunction within the vacuum than unconstrained motion. The new system is now used in the Toulouse Planeterrella and reveals new phenomena depending on the distance between the spheres. The two magnets have strength of $400 \mathrm{mT}$. In the future, we will determine the lowest usable magnetic intensity and examine its impact on the observed phenomenon.

\subsection{Modelling}

In order to make the experiment more understandable to a wider audience, we have built a numerical model to simulate the trajectories of the particles and to map the emission regions. This model is based on a Monte Carlo technique. The location, direction and velocity of the particles injected into the vacuum are decided randomly. The trajectory is then calculated by solving the equation of motion using an adaptative step Runge Kutta 45 method. Because of the Debye screening effect, we consider that the electric field is fully screened within a very small region close to the cathode. The width of this region has been calculated in Lilensten et al. (2009), to be equal to approximately $3 \mathrm{~mm}$. This means that the particles are considered to leave the cathode with a velocity remaining constant beyond the Debye sphere and are then only affected by the magnetic field. This field is considered to be the superposition of the two dipolar fields created by the magnets in the spheres. Elastic cross sections are considered with an isotropic angle phase function and some inelastic processes are used to obtain a rough evaluation of the emissions. In particular, the secondary electrons are not yet considered.

The code contains a module to visualize individual trajectories. An example of the computed trajectories is shown in Figure 8. Currently, the code does not reproduce the light emission regions. In further development, we will introduce a realistic electric field by computing a more precise electric field 


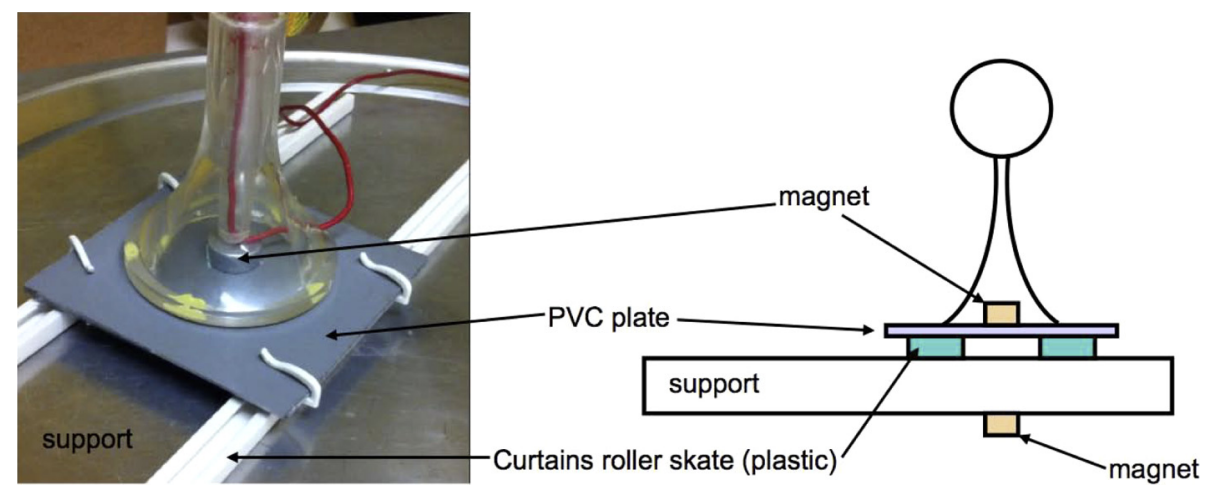

Fig. 7. View of the low-cost system designed by the students of the technological institute in the University of Toulouse 3 . The small sphere is fixed on a PVC plate, which moves on "curtain rails". Two magnets located inside and outside the vacuum chamber make the movement.

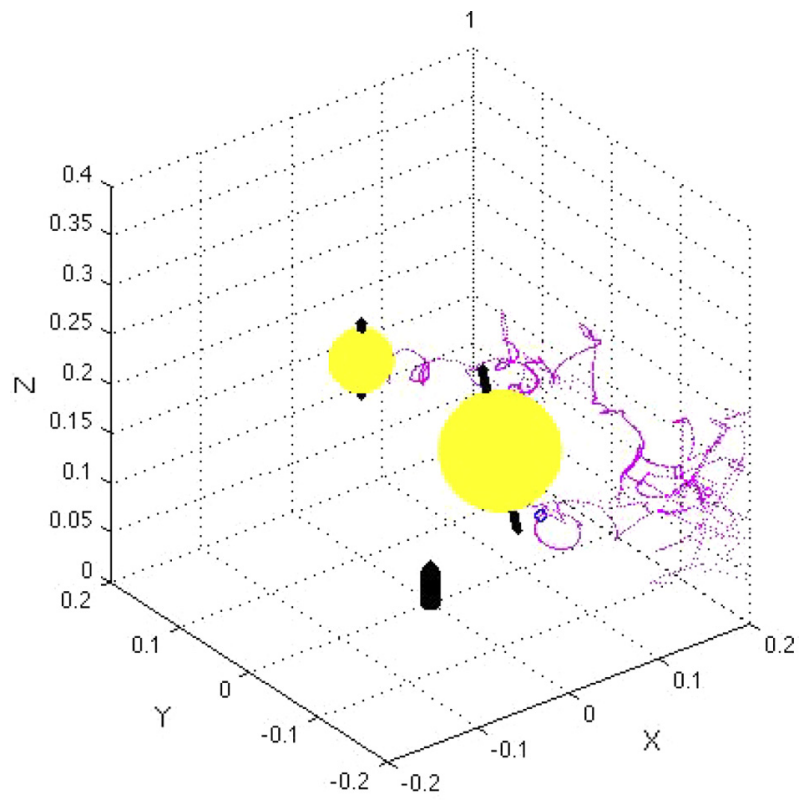

Fig. 8. Simulation of the trajectory of a particle with an initial energy of $67 \mathrm{eV}$. In this example, only elastic collisions are taken into account. Each dot in the trajectory represents a collision. The two spheres are shown in yellow, with the magnetic axis in black.

map. Depending on the level of education of the audience, several phenomena can be illustrated. For postgraduate or masters level students, the experiment and this model can be used for a plasma physics practical work, for example to confirm or refute the validity of the guiding centre approximation.

\subsection{Artistic}

Many artists are fascinated by the auroras. The Planeterrella website has been a source of inspiration for paintings, installations and movies in several countries, such as France, Belgium and Germany. The Planeterrella has been displayed at exhibits such as the "Night of the Castles" where it was shown as a work of art. Italian sculptors recently claimed that the Planeterrella was not about science but about sculpting light. A theatre company preparing a production on Birkeland currently uses the Planeterrella. Here the Planeterrella will be on the stage as one of the actors. The artists have asked to introduce different gases into the vacuum chamber in order to vary the colours of the aurora. This is possible using the Planeterrellas II and III.
Art can therefore be an important inspiration when showcasing scientific outreach experiments.

\subsection{Popularization of space weather, popularization of science}

The Planeterrella can be used to visualize the Van Allen Radiation Belts, the auroral ovals, the solar corona, the polar cusp, the magnetopause, and to examine the links between space weather and Earth space environment. The experiment allows one to demonstrate where and why spacecraft are exposed to space weather hazards. One of the most frequently asked questions concerns the link between space weather and climate. Although this is a highly controversial topic and no definitive answer can be given, the Planeterrella does at least allow one to expound on some of the mechanisms by which solar activity impacts our upper atmosphere.

Politicians (mayors, deputies, etc.) constitute an important target audience. As they tend to be very busy, information must be imparted to them in a focused and succinct manner. In this regard the Planeterrella has proven to be an efficient tool.

Finally we consider the visitor experience of the Planeterrella's audience. The UK group monitored the Planeterella's audience. Though they did not have the resources to perform a visitor analysis of all tens of thousands visitors, a focus group of school pupils at one school was created and asked to fill in a questionnaire. Of the respondents $100 \%$ either strongly agreed or agreed with the statement "I found the presentation interesting", $100 \%$ either strongly agreed or agreed with the statement "Science affects everyday life" and 75\% agreed with the statement "I would like to find out more about careers in science".

\section{The limitation of the Planeterrella}

The Planeterrella has its origins in Birkeland's work. It must first and foremost be considered as an outreach experiment and a simulator. No simulation - numerical or experimental is the truth. Several of the phenomena that are shown more resemble analogies than the reality of the planetary space environments. For example, there are only electrons and no protons in the wind produced, there is no magnetosphere, no magnetospheric currents or particle acceleration. There is no atmospheric density gradient either. Therefore, it is very important that experimenters do not confuse the public but clearly state that some of the simulated phenomena do not accurately reflect 
the reality of space. Other features are closer to reality, such as the making of the radiation belt, of the auroral ovals, of the cusps, and the physics behind the auroral emissions.

\section{Conclusion}

The Planeterrella has become an extremely popular and successful public outreach experiment, fascinating many tens of thousands of visitors from the general public to professional scientists. The Planeterrella received the first international prize for outreach activities from the Europlanet Framework 7 program in 2010 and the French Ministry of Science outreach prize "Le goût des sciences" in November 2012. Below is a summary of the main aspects of the project and how they have contributed to its success:

(a) The plans are given freely to any public institute. This constitutes a saving of several thousand Euros. The plans are not patented; instead a Gentleman's Agreement has to be signed. This is a political choice; public science is costly but the outcomes of public science should remain free for the public. Thus the Planeterrella inspires a culture of trust and cooperation that has contributed to its success.

(b) Science benefits by being widely disseminated and shared. To that extent, Europe has developed several networking tools. This is one of the key factors that make Europe attractive and dynamic within different fields of research. Amongst the tools, COST was very effective in contributing to the international development of the Planeterrella.

(c) Most modern "interactive" scientific exhibitions rely on computers, for example buttons to press in order to get an effect from a robot or a new figure on a screen. Such exhibits are certainly attractive to children. Compared to such experiments, the Planeterrella looks like a 19th century experiment. However, in the eyes of the visitors, this looks new, different from what they are used to and, surprisingly, very modern.

(d) The Planeterrella is very cost-efficient. One Planeterrella represents a gross investment of EUR 8000. There are now seven completed Planeterrellas and more in preparation. Live auroral demonstrations have been presented to about 50000 people (and many more have seen the exhibits on TV). This represents a cost of less than EUR 0.9 per visitor. This cost will decrease further in the future when more Planeterrellas are completed, including one in the largest scientific museum in Paris (le Palais de la Découverte), which welcomes several tens of thousands of visitors each year. Thus we consider the relatively small investment in the Planeterrellas to be a worthwhile and cost-efficient expenditure.

(e) The post-evaluation process of the Planeterrella project has proven to be very important when obtaining funding. This has certainly been true in France, where money can be diverted from other projects and the reviewers evaluate the use of the money at the end of the project. This way is opposite to the call-and-selection process at use in many other countries.

Acknowledgements. Amongst the contributors to the Planeterrella project, JL especially thanks Philippe Jeanjacquot, System 2.0 as well as Olivier Brissaud, Philippe Chauvin, Karine Briand, Herve Lamy, Laurent Lamy, Baptiste Cecconi, Jean-Francois Donati. Several $\mathrm{PhD}$ students were and still are a wonderful support, including Gael Cessateur, Hélène Ménager, David Bernard and of course the enthusiastic, imaginative and always supportive Guillaume Gronoff. The Alpes Délégation CNRS, the Université Joseph Fourier, the IPAG laboratory and the PNST administrations are also thanked for their constant support. The Planeterrella has been built at the SERAS / CNRS workshop. The COST ES 0803 community has also supported this project enthusiastically. More than 40 students have already worked on the project for their studies. Amongst them, we thank particularly Camille Patris, Arthur Birac and Marie Garcon. CSW and PV are indebted to Eddy Equeter and Johan De Keyser (BIRA-IASB) for their unfailing involvement, work, and ideas in the current implementation of the Belgian Planeterrella.

The coordination part of this project is funded under the European project ESPAS 7th FP RI 283676.

\section{References}

Brundtland, T., The Laboratory Work of Professor Birkeland in The Auroral Observatory, University of Tromsø, Tromsø, Norway, 1997.

Egeland, A., and J. Burke, Birkeland, the first space scientist, Astrophysics and Space Science Library, Vol. 325, Springer, Dordrecht, 2005.

Messerotti, M., R. Baccomi, D. Iugovaz, and J. Lilensten, A Terrella device for simulating aurora-like phenomena in a box, Earth Moon Planet, Springer Science + Business Media B.V, DOI: 10.1007/s11038-008-9262-y, 2008.

Lilensten, J., M. Barthélémy, C. Simon, P. Jeanjacquot, and G. Gronoff, The Planeterrella, a pedagogic experiment in planetology and plasma physics, Acta Geophys., 57 (1), 220-235, DOI: 10.2478/s11600-008-0079-x, 2009.

Gronoff, G., and C. Simon-Wedlund, Auroral formation and plasma interaction between magnetized objects simulated with the Planeterrella, IEEE Trans. Plasma Sci., 39, 2712-2713, 2011.

Cite this article as: Lilensten J, Provan G, Grimald S, Brekke A, Flückiger E, Vanlommel P, Wedlund CS, Barthélémy M \& Garnier P: The Planeterrella experiment: from individual initiativeto networking. J. Space Weather Space Clim., 2013,3 , A07. 